\title{
Optimizing the DVB-SH FEC Scheme for Efficient Erasure Recovery
}

\author{
Mohamed Amine Ismail \\ R\&D Department of UDcast \\ Sophia Antipolis, France \\ Email: Amine.Ismail@udcast.com
}

\author{
Thierry Turletti \\ INRIA, PLANETE Team \\ Sophia Antipolis, France \\ Email: Thierry.Turletti@inria.fr
}

\author{
Walid Dabbous \\ INRIA, PLANETE Team \\ Sophia Antipolis, France \\ Email: Walid.Dabbous@inria.fr
}

\begin{abstract}
DVB-SH is a new broadcasting standard offering a mobile TV service for handheld devices using hybrid satellite/terrestrial-repeaters solution. A new link layer protection algorithm called Multi-Burst Sliding Encoding (MBSE) has been recently adopted to cope with the long fading time introduced by the direct satellite link. This paper presents a method to optimize the MBSE parameters and an analysis of the performance gain. Various sets of parameters are studied and optimized with respect to the link performance. Furthemore, an algorithm was provided to compute the optimum values of the MBSE parameters according to some constraints. we have implemented MBSE in two UDcast DVB-SH equipements and we have validated our optimization method using intensive experiments with typical usage scenarios under a hardware-emulated wireless link.
\end{abstract}

\section{INTRODUCTION}

The recent developments and improvements of the broadcasting techniques and video compression had a big impact on the television market. Beside classical TV, people are willing to watch their favorite program on their mobile devices anytime, anywhere. In the last few years, several standards broadcast mobile TV have emerged. DVB-H [1], [2], Digital Video Broadcasting-Handheld, one of the most prevalent mobile TV formats, is based on DVB-T [3] ( $\mathrm{T}$ for Terrestrial) but takes into account the limited battery capacity of handheld devices. To solve the problem of power consumption, a time division multiplex transmission is introduced at the link layer level. This technique is called Time-Slicing [4]. The TimeSlicing is one of the innovations of DVB-H. The key idea is to transmit data in "bursts" exclusively carrying a single service. The data rate of bursts is much higher compared to the average data rate of the service itself; typically a burst uses the whole channel rate during its transmission. This enables a time selective reception of the desired service by switching to a power-save mode during the transmission of other services.

DVB-SH [5] ( $\mathrm{SH}$ for Satellite services to Handhelds) is an independent derivative of DVB-H. It was designed for frequencies below $3 \mathrm{GHz}$, supporting UHF band, L-Band or S-band. Like its predecessor specification (DVB-H), it is based on DVB IP Datacast (IPDC) [6] delivery and electronic service guides (ESG) [7]. The DVB-SH standard defines the way to deliver "IP based media content and data" to handheld terminals using hybrid satellite/terrestrial solution. It employs a high-power geo-stationary satellite for cost-effective nationwide coverage and a network of low-power repeaters to provide urban and indoor coverage. The satellite ensures nationwide direct reception of up to nine channels at 256 kbits/s. Repeaters in urban areas retransmit the received data at the frequency of the satellite carrier and therefore are able to offer indoor coverage. Synchronization between the terrestrial repeaters and the satellite allows the receiver to see the satellite signal as a simple echo of the terrestrial repeater signal $^{1}[8]$. To increase the system capacity in urban areas, adjacent carrier transmitters complement the satellite signal, allowing the transmission of additional channels.

DVB-H key technologies such as Orthogonal Frequency Division Multiplexing (OFDM) modulation, time slicing and IP data casting are maintained while an improved link budget is achieved by employing turbo-codes. Furthermore, significant improvements are made within the terminals (higher antenna gain and reception diversity).

One of the major challenging issue addressed by DVB$\mathrm{SH}$ is the protection of data against the impairments of the direct satellite link which is often modeled using a Land Mobile Satellite [9] (LMS) model ${ }^{2}$. This kind of channel is characterized by long fading time where the signal can be interrupted for several seconds due to physical obstacles like tree, tunnel or weather conditions. To cope with this problem, a new encoding algorithm called Multi Burst Sliding Encoding (MBSE) has been adopted. This algorithm is able of generating an outer FEC to recover several consecutive lost bursts. It extends the DVB-H intra-burst MPE-FEC (MultiProtocol Encapsulation-Forward Error Correction)[4] protection to an inter-burst protection so that complete burst losses could be recovered while taking into account the specificity of mobile handheld devices.

There are four main parameters that regulate the operation of MBSE: The encoding parallelization factor $(B)$, The FEC spreading factor $(S)$, the number of computed outer FEC columns from an encoding matrix $\left(F_{o}\right)$ and the number of

\footnotetext{
${ }^{1}$ Single Frequency Network (SFN) operation comes from the robustness of the transmission system against echos. In fact echos can be passively generated by the environment (reflection, refraction, etc. in short, multipath propagations) or be artificially produced by active transmitters radiating on the same frequency, at the same time, the same bits.

${ }^{2}$ In satellite to mobile communications, there is generally a strong line-ofsight component in addition to the diffuse multipath component. The spectral and statistical properties of both components are influenced by shadowing caused by obstacles such as trees, houses or small buildings.
} 
rows of an encoding matrix $\left(N_{R}\right)$. However according to the maximum end-to-end delay tolerated by the application layer, constraints on the memory usage and the service bitrate, many combinations of these parameters, resulting in a very different performance, can be used. The DVB-SH standard does not give a method to determine the best set of these parameters to be used. In this paper we first evaluate theoretically the performance of MBSE, then we derivate a linear problem allowing the computation of the optimum values of $B$ and $S$ and $N_{R}$ according to the FEC ratio and the end-to-end delay. Finally we evaluate the performance of MBSE under an LMS and TU6 (Terrestrial Urban-6 paths) ${ }^{3}[10]$ channels while varying $B, S, N_{R}, F_{o}$ and the end-to-end delay, and we demonstrate that our method for the parameters computation provides the optimum values. To our knoweledge, this is the first work focusing on the optimization of the DVB-SH MBSE parameters. A similar work [11] has been performed for the DVB-H FEC scheme. In [11] results are only obtained by simulations using a very simple channel estimation while in our work, the results are obtained theoritically and then by experiments using a real implementation of the standard and a hardware channel emulator. Furthemore the DVB-H and DVBSH FEC schemes are quite different.

The rest of the paper is organized as follows: section II describes the Multi-burst Sliding Encoding Algorithm. Then a theoretical analysis of its recovering capacity is presented in section III and an algorithm is provided to compute the optimum values of $B, S$ and $N_{R}$. In section IV we evaluate the performance of MBSE applied to the RS $(191,255)$ under an LMS and TU6 channel model while varying the parameters that regulate the operation of the algorithm and we demonstrate by experiments the correctness of our analysis given in section III. Finally section V presents our conclusion and future work.

\section{The Multi Burst Sliding EnCoding}

The Multi Burst Sliding Encoding mechanism is a link layer solution for extending the duration of the protection while still using small size blocks. It extends the existing DVB$\mathrm{H}$ MPE-FEC intra-burst protection scheme to an inter-burst protection so that complete burst losses may be recovered by producing an "outer/inter FEC" in a regular basis (a fraction of the protection is created every burst). In order to achieve this protection, data coming from several bursts are interleaved inside parallel matrices before FEC protection is applied, even if original data are not sent interleaved eventually. On the reception side, received data are also interleaved before being placed in decoding matrices. In order to achieve this extension the following parameters are needed:

- $B$ : The encoding parallelization factor expressed in encoding matrix units. Each burst is split into $B$ parts distributed over $B$ encoding matrices.

- $S$ : The depth of the FEC spreading factor: The generated FEC is spread over $S$ bursts.

\footnotetext{
${ }^{3}$ TU6 models the terrestrial propagation in an urban area. It uses 6 resolvable paths, each one characterized by a delay, a power, and fiding model.
}

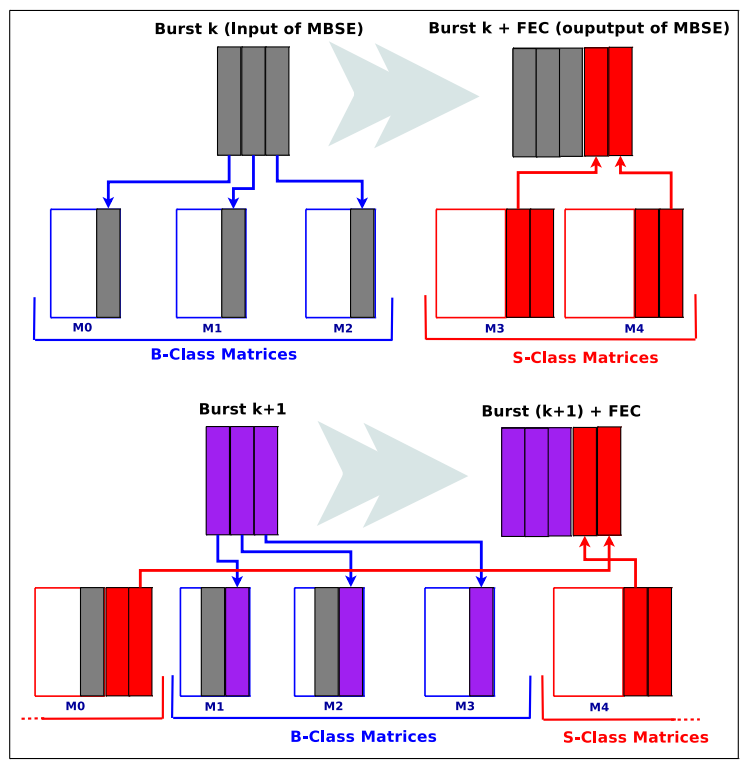

Figure 1. MBSE Encoder

- $F_{o}$ : Number of computed outer-FEC columns from an encoding matrix.

The algorithm works as follows: The encoder allocates a set of $(B+S)$ encoding matrices divided into two classes : $B$-class made of $B$ matrices and $S$-class made of $S$ matrices. At each iteration a sliding window (hence the name of the algorithm) is applied to the $(B+S)$ matrices and thus one matrix from the $B$-class goes to the $S$-class and vice versa.

Each data burst (sevral IP datagrams) is mapped ${ }^{4}$ in an Application Data Table (ADT) (matrix of bytes) made of $C$ columns and $N_{R}$ rows. Each ADT is divided into $B$ parts made of a number of non contiguous columns taken in a particular way. Each part is placed in a specific encoding matrix from the $B$-class. At each iteration, only one specific encoding matrix - from the $S$-class - containing columns coming from several bursts is encoded and thus, $F_{o}$ FEC columns are generated. Each IP datagram from the burst is encapsulated in an MPE section $^{5}$ and sent without additional delay. At the end of the burst, some FEC columns ${ }^{6}$, chosen in a specific way from the $S$-class matrices, are encapsulated in an MPE-FEC sections ${ }^{7}$ and sent.

Figure 1 shows a simple example of an encoder with $B=3$, $S=2$ and $F o=2$. We suppose that each burst can be mapped on an $A D T$ with 3 columns.

With MBSE, in order to perform the decoding operation, the mobile device has to wait until the reception of all the IP datagrams and the parity symbols of each encoding matrix. Parity symbols generated from one encoding matrix are sent

\footnotetext{
${ }^{4}$ The ADT is filled with the bytes of the IP datagarms

${ }^{5}$ An MPE section consists in a header followed by an IP datagram and a CRC32

${ }^{6}$ The number of FEC columns per burst depends on the FEC ratio and the burst size

${ }^{7}$ An MPE-FEC section consists in a header followed by several FEC bytes and a CRC32
} 
into $S$ time-sliced bursts. The terminal has first to receive the $B$ bursts necessary to retrieve all the IP datagrams, and then the $S$ bursts necessary to retrieve the FEC. Because of this, the mobile device has to wait a total of $(B+S)$ bursts to be able to fill one encoding matrix and achieve full protection. As the mobile device has to buffer $(B+S)$ bursts, the size of the required required buffer is equal to $($ BurstSize $*(B+S))$.

\section{A. Encoder algorithm}

\section{Initialization}

The encoder allocates $(B+S)$ encoding matrices $\left\{M_{0}, \ldots, M_{(B+S-1)}\right\}$ with $C$ columns and $T$ rows each. Each encoding matrix $M_{i}$ is composed of two parts: The application data table $M A D T_{i}$ and FEC data table $M F D T_{i}$.

The following process is applied for each burst of data numbered $k$.

Step 1: Processing and transmission of the $k^{t h}$ data burst.

- Map the data burst in an $A D T$ noted $A D T_{k}$.

- Send the data burst as is.

- Create a FEC burst containing $F_{o}$ FEC columns $\left\{F_{0}, \ldots F_{F o-1}\right\}$ extracted from $S$ interleaved $M F D T$ $\left\{M F D T_{f(0, k)}, \ldots, M F D T_{f(F o-1, k)}\right\}$ among $B+S . F_{i}$ is the $i^{\text {th }}$ FEC column of the $M F D T_{f(i, k)}$. The $f$ function is defined as:

$$
f(j, k)=(k-1-(j \bmod S)) \bmod (B+S)
$$

Step 2: Interleaving of the $A D T_{k}$ (the $k^{\text {th }}$ data burst) and outer FEC computation.

Interleave the $A D T_{k}$ inside $B \quad M A D T$ $\left\{M A D T_{g(0, k)}, \ldots, M A D T_{g(C, k)}\right\}$ among $B+S$ such as the $i^{t h}$ column of the $A D T_{k}\left(C_{i, k}\right)$ is copied into the $p(i)^{t h}$ column of the $M A D T_{g(i, k)}$. The $g$ and $p$ functions are defined as:

$$
\begin{aligned}
& g(i, k)=(k+(i \bmod B)) \bmod (B+S) \\
& p(i) \quad=(B-1-(i \bmod B)) \times\left\lfloor\frac{C}{B}\right\rfloor+ \\
& \max (0 ;(C \bmod B)-(i \bmod B)-1)+\left\lfloor\frac{i}{B}\right\rfloor
\end{aligned}
$$

- Encode the Matrix $M_{h(k)}$. The FEC encoding function generates the $M F D T_{h(k)}$ from the $M A D T_{h(k)}$. The $h$ function is defined as:

$$
h(k)=k \bmod (B+S)
$$

\section{B. Decoder algorithm}

The decoding operation is basically the reverse of the encoding one. When a data burst $k$ is received, it is interleaved inside $B$ matrices and the outer FEC carried by this burst is inserted in the corresponding matrices. The encoding matrix $M_{u(k)}$ is decoded, then de-interleaved and finally the burst $l(k)$ can be retrieved and passed to the upper layer. The $u$ and $l$ functions are defined as follows:

$$
\begin{gathered}
u(k)=(k+B) \bmod (B+S) \\
l(k)=(k-B-S+1) \bmod (B+S)
\end{gathered}
$$

\section{Performance Analysis}

In this section, we derive an expression of the burst recovery capacity of the MBSE scheme.

A burst $k$ is made of $C$ data columns mapped into an $A D T$, and $F_{o}$ outer FEC columns. In order to recover a lost burst $k$, all the encoding matrices containing a portion of that burst must be successfully decoded (all erasure recovered). At the encoder, $A D T_{k}$ is interleaved with the $(B-1)$ previous $A D T\left\{A D T_{k-(B-1)}, A D T_{k-(B-2)}, \ldots, A D T_{k-1}\right\}$ and with the $(B-1)$ following $A D T$ $\left\{A D T_{k+1}, A D T_{k+2}, \ldots, A D T_{k+(B-1)}\right\}$ inside $B$ encoding matrices $\left\{M_{h(k)}, M_{h(k+1)}, \ldots, M_{h(k+B-1)}\right\}$. Each matrix contains $\frac{C}{B}$ data columns coming from $A D T_{k}$. In the case of a "perfect FEC code", the decoder needs to receive $\frac{C}{B}$ FEC columns for each encoding matrix $M_{h(k)}$. This makes sense only if $\frac{C}{B} \leq F_{o}$. On the other hand, the FEC columns produced by the encoding of the matrix $M_{h(k)}$ are spread over the $S$ following bursts $\left\{\right.$ Burst $_{k+1}$, Burst $_{k+2}, \ldots$, Burst $\left._{k+S}\right\}$. Burst $_{k+i}$ contains $\frac{F_{o}}{S}$ FEC columns coming from the encoding matrix $M_{h(k)}$. Therefore, to retrieve the burst $k$, the decoder needs to receive the $(B-1)$ previous bursts and the $\left\lceil B-1+\frac{C . S}{B . F_{o}}\right\rceil$ following bursts.

In a more general case, when $n$ successive bursts $\left\{A D T_{k}, A D T_{k+1}, \ldots, A D T_{k+n-1}\right\}$ are lost, the set $\chi$ of the involved encoding matrices is defined as:

$$
\begin{aligned}
\chi= & \left\{M_{h(k)}, M_{h(k+1)}, \ldots, M_{h(k+\min (B ; n)-1)},\right. \\
& M_{h(k+\min (B ; n))}, \ldots, M_{h(k+\min (B ; n)-1),} \\
& \left.M_{h(k+\max (B ; n))}, \ldots, M_{h(k+n+B-2)}\right\}
\end{aligned}
$$

To show more clearly the calculations, we divide the set $\chi$ into three subsets $\chi_{1}, \chi_{2}$ and $\chi_{3}$ such that:

$$
\begin{aligned}
& \chi_{1}=\left\{M_{h(k)}, M_{h(k+1)}, \ldots, M_{h(k+\min (B ; n)-1)}\right\} \\
& \chi_{2}=\left\{M_{h(k+\min (B ; n))}, \ldots, M_{h(k+\max (B ; n)-1)}\right\} \\
& \chi_{3}=\left\{M_{h(k+\max (B ; n))}, \ldots, M_{h(k+n+B-2)}\right\} \\
& \chi=\chi_{1} \cup \chi_{2} \cup \chi_{3}
\end{aligned}
$$

To decode the matrices of $\chi_{1}$, the set of bursts $\psi_{1}$ must be received by the decoder and the constraint $C_{1}$ must be satisfied. $\psi_{1}$ and $C_{1}$ are defined as:

$$
\psi_{1}=\left\{\text { Burst }_{k+n}, \ldots, \text { Burst }_{k+m_{1}}\right\}
$$




$$
\begin{aligned}
& C_{1}: \quad\left\lceil n+(j+1) \frac{C . S}{B . F_{o}}-1\right\rceil \leq j+S \\
& \forall j \mid 0 \leq j \leq \min (B ; n)-1 \\
& m_{1}=\quad\left\lceil n+\min (B ; n) \times \frac{C . S}{B . F_{o}}-1\right\rceil
\end{aligned}
$$

To decode the matrices of $\chi_{2}$, the set of the bursts $\psi_{2}$ must be received by the decoder and the constraint $C_{2}$ must be satisfied. $\psi_{2}$ and $C_{2}$ are defined as:

$$
\begin{gathered}
\left.\psi_{2}=\quad \text { Burst }_{k+n}, \ldots \text { Burst }_{k+m_{2}}\right\} \\
C_{2}: \quad\lceil n-\min (B+j+1 ; n)+ \\
\left.\quad+\min (B ; n) \frac{C . S}{B . F_{o}}-1\right\rceil \leq S \\
\forall j|0 \leq j \leq| n-B \mid-1 \\
m_{2}=\quad\left\lceil\max (B ; n)+\min (B ; n) \times \frac{C . S}{B . F_{o}}-1\right\rceil
\end{gathered}
$$

To decode the matrices of $\chi_{3}$, the set of the bursts $\psi_{3}$ must be received by the decoder and the constraint $C_{3}$ must be satisfied. $\psi_{3}$ and $C_{3}$ are defined by:

$$
\begin{aligned}
& \psi_{3}=\left\{\text { Burst }_{k+\max (B ; n)+1}, \ldots, \text { Burst }_{k+m_{3}}\right\} \\
& C_{3} \quad: \quad\left\lceil(\min (n ; B)-1) \frac{C}{B \cdot F_{o}}\right\rceil \leq 1 \\
& m_{3}=\left\lceil\max (B ; n)+(\min (B ; n)-1) \times \frac{C \cdot S}{B \cdot F_{o}}\right. \\
& \left.+(\min (B ; n)-2) \times\left(1-\frac{C . S}{B \cdot F_{o}}\right)\right]
\end{aligned}
$$

Therefore, to decode all matrices of $\chi$, the set of the bursts $\psi=\psi_{1} \cup \psi_{2} \cup \psi_{3}$ must be received by the decoder and the constraint $C$ (satisfying $C_{1}, C_{2}$ and $C_{3}$ ) must be satisfied. $\psi$ and $C$ are defined by:

$$
\begin{aligned}
& \psi=\left\{\text { Burst }_{k+n}, \ldots, \text { Burst }_{k+m}\right\} \\
& C: \quad\left\lceil n-\min (B ; n) \times\left(1-\max \left(\frac{C \cdot S}{B \cdot F_{o}} ; 1\right)\right)\right\rceil \leq S \\
& m=\quad\left\lceil\max (B ; n)+\frac{C . S}{B \cdot F_{o}}-1+\right. \\
& \left.\max \left(\frac{C . S}{B . F_{o}} ; 1\right) \times(\min (B ; n)-1)\right\rceil
\end{aligned}
$$

From (19), we can see that when $\frac{C . S}{B \cdot F_{o}} \leq 1$, the maximum recovery capacity of the MBSE algorithm becomes $S$ successive bursts. On the other hand, when $\frac{C . S}{B \cdot F_{o}} \leq 1$ then $C . S \leq B . F_{o}$. since $B$ is equal to $M-S, S \leq \frac{M}{\left(\frac{C}{F_{o}}+1\right)}$. This means that MBSE is able to recover up to $\frac{M}{\left(\frac{C}{F_{o}}+1\right)}$ consecutive lost bursts. For large enough FEC percentage $\left(F_{p}=\frac{F_{o}}{C}\right)$, This expression ( bounded by $\mathrm{M}$ ), can reach $(M-1)$.

To recover the $n$ successive lost bursts, the decoder needs to receive the bursts from $k+n$ to $k+m$. When condition $C$ is satisfied, we obtain $m-n \leq B+S$. Therefore the optimum values of $B$ and $S$ for a given FEC percentage $\left(F_{p}=\frac{F_{o}}{C}\right)$ and

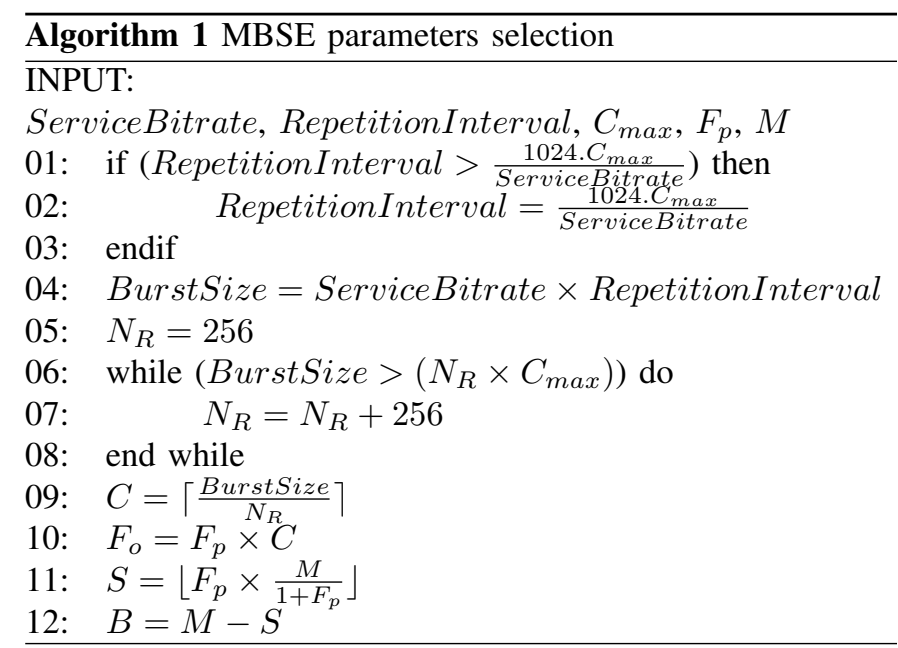

a fixed value of $B+S$ are computed by solving the following linear program:

$$
\begin{array}{ll}
\text { Maximize } & S \\
\text { Subject to } & \left\{\begin{array}{l}
\frac{C . S}{B \cdot F_{o}} \leq 1 \\
B+S=M
\end{array}\right.
\end{array}
$$

Problem 21 has the following closed-form solution:

$$
\begin{gathered}
S=\left\lfloor F_{p} \times \frac{M}{1+F_{p}}\right\rfloor \\
B=\left\lceil\frac{M}{1+F_{p}}\right\rceil
\end{gathered}
$$

On the other hand, the number of rows has an impact on the performance of MBSE. In fact, even if the size of an IP datagram is independent from the value of $N_{R}$, the payload of a FEC section consists in a column of bytes taken from an $M F D T_{i}$. Thus its size is equal to the value of $N_{R}$. So what is the impact of the section size on the packet loss rate? Let $p$ the byte error probability, and $T$ the section size, then the section error probability is:

$$
\sum_{n=1}^{T}\left(\begin{array}{c}
T \\
n
\end{array}\right) \cdot p=1-(1-p)^{T} \approx T \cdot p
$$

approximation being valid whenever $p \ll 1$. From equation 23 we can see that the section error probability grows linearly with the section size. the higher the section size the higher the probability to have at least one erroneous byte.

As shown previously, the performance of MBSE depends on several parameters which are interdependent. Different combinations of these parameters may result in a deviation of the recovery performance. Algorithm 1 can be used to compute the optimum values for the number of rows $N_{R}, B$ and $S$ according to a service bitrate (ServiceBitrate), repetition interval (RepetitionInterval derived from the power saving ratio), the maximum number of columns per ADT $\left(C_{\max }\right)$, the FEC percentage $\left(F_{p}\right)$ and $M$ (derived from the end-to-end delay or the memory size of the receiver device). 


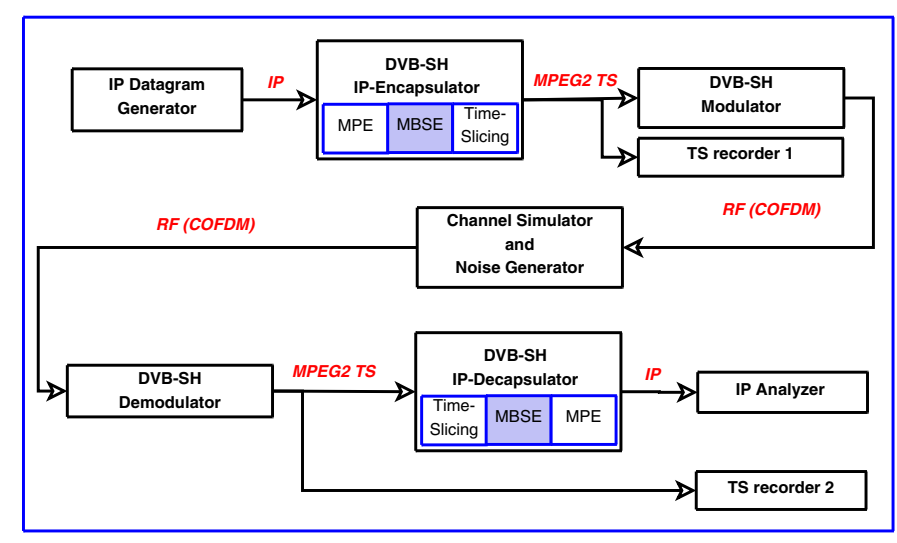

Figure 2. DVB-SH Test bed

\section{Experimental Performance of the Sliding ENCODING SCHEME}

In the following we applied the RS(191,255) MBSE algorithm to a live stream while varying the values of $B, S$, $N_{R}$, and $F_{o}$. The aim is to find the best combination of these parameters that maximizes the recovering capacity of the algorithm.

1) Test bed description: We have implemented the encoding and decoding algorithms of the MBSE in two standard UDcast [12] equipments: the DVB-SH IP-Encapsulator and the DVB-SH Navigator.

For experimental purpose the test bed described in Figure 2 is set up. The MPE and MPE-FEC sections are generated so that an IP datagram forms the payload of an MPE section and an outer-FEC column forms the payload of an MPEFEC section [13]. The MPE and MPE-FEC sections are then fragmented into MPEG2 [14] packets carried through an ASI cable to a DVB-SH OFDM modulator. The modulated signal is passed through a hardware channel emulator [15] implementing the TU6 and the LMS models. The Noisy signal is demodulated by the DVB-SH signal demodulator and the generated MPEG2 packets are passed to the MBSE decoder to retrieve the IP datagrams. An IP analyzer then provides statistics on the packet loss rate (PLR) and the Erroneous Second Ratio (ESR $)^{8}$. The following parameters are used in the experiments :

- Repetition interval: $1 \mathrm{~s}$

- Physical layer FEC ratio: 50\%

- Physical layer interleaving: $100 \mathrm{~ms}$

- Physical modulation: QPSK

- Mobile speed: $3 \mathrm{Km} / \mathrm{h}$

2) Experiments Results: In the first experiment we examine the effect of different values of number of rows $\left(N_{r}\right)$ against erroneous second ratio over a TU6 and LMS channels while keeping the service bitrate and the repetition interval constant. Figures 3 and 4 report the error free second ratio $(E F S R=$ $1-E S R)$ after applying MBSE to a noisy signal under a TU6

\footnotetext{
${ }^{8}$ ESR represents the ratio between the number of seconds containing a least one erroneous IP datagram and the duration of the experiment
}

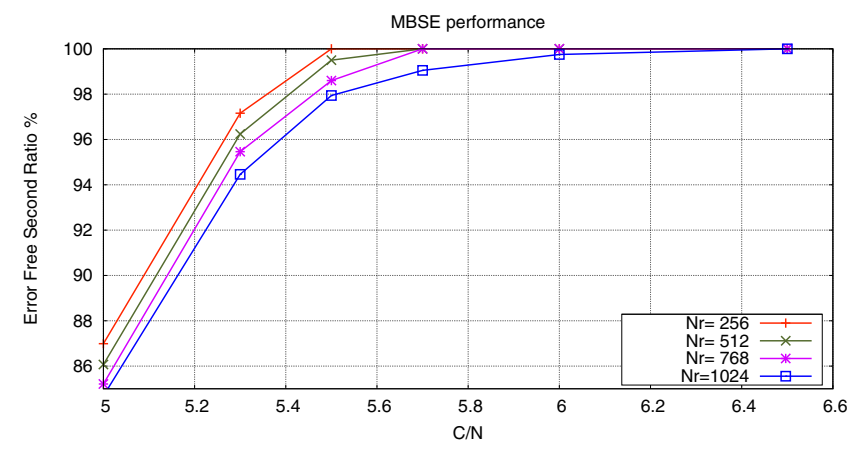

Figure 3. Error Free Second Ratio in TU6 channel while varying the C/N for different values of number of rows

and LMS channels respectively, while taking different values of $N_{R}$. From these set of curves we can clearly see that the lower the value of $N_{R}$ parameter the better the performance of MBSE for both channel models. For example, in a TU6 channel an EFSR of $100 \%$ is reached at $5.5 \mathrm{db}$ by using a value of $N_{R}$ equal to 256, while the same EFSR is only reached at $6.5 \mathrm{db}$ if we use 1024 rows per matrix.

The second experiment consists in finding the optimum combination of $B$ and $S$ that gives the best performance of MBSE according to a given FEC ratio and the maximum endto-end delay $(M=B+S)$. the values of $N_{R}$ and $C$ have been fixed to 1024 rows and 100 columns respectively. The carrier to noise ratio $(\mathrm{C} / \mathrm{N})$ has been fixed to $5.4 \mathrm{db}$. Figures 5,6 and 7 report the packet loss rate (PLR) under an LMS channel while varying $B$ and $S$ for different values of FEC ratio and endto-end delay $(M)$. From these set of curves, we can observe that for each couple of $(B, S)$ we obtain a different value of PLR even if we keep the FEC ratio, $M=(B+S)$ and the the $\mathrm{C} / \mathrm{N}$ constant. For the nine curves, the gap between the worst and the best PLR is higher than 5\%. For example for $\mathrm{M}=16$ and a FEC ratio of $33 \%$, the worst PLR is about $\sim 12 \%$ (for $B=1$ ) and the best one is about $\sim 4 \%$ (for $B=11$ ). We can also check that the minimum PLR is always obtained for the values of $B$ and $S$ that verify the eq. 21. For example: for $\mathrm{M}=10$ and FEC ratio of $33 \%$ the optimum value of $(B, S)$ is $(7,3)$.

As demonstrated by experiments, the parameters regulating the operation of MBSE have an impact on the quality of service in term of packet loss rate. Indeed, comparing to the worst case, we can achieve a gain of $\sim 1 \mathrm{db}$ by setting the appropriate combination of these parameters. This gain or optimization in term of $\mathrm{C} / \mathrm{N}$ can be used by the broadcasters to increase the coverage area of the transmitters or to reduce the transmission power of the base stations while maintaining the same coverage and the same quality of service.

\section{CONCLUSION}

The DVB-SH standard defines an error correction scheme called Multi-Burst Sliding Encoding (or MBSE) for the link layer to combat effects of the LMS channel model. However, the parameters configuration of MBSE is left open. In this paper, The burst interleaving scheme of MBSE has been 


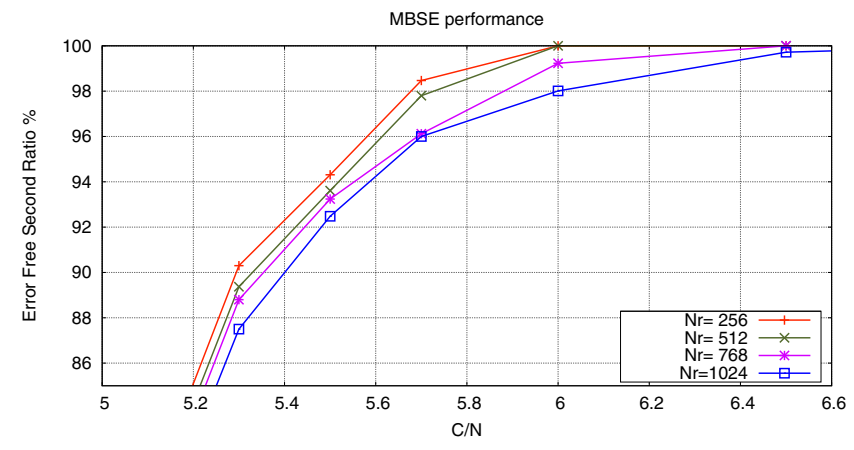

Figure 4. Error Free Second Ratio in LMS channel while varying the C/N for different values of number of rows

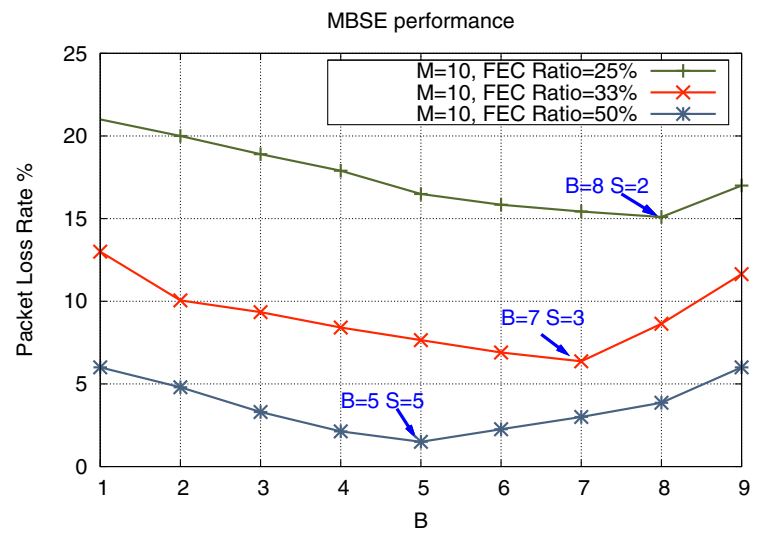

Figure 5. Packet Loss Rate in LMS channel while varying $B$ and $S$ subject to $B+S=10$ for a FEC ratio of $25 \%, 33 \%$ and $50 \%$

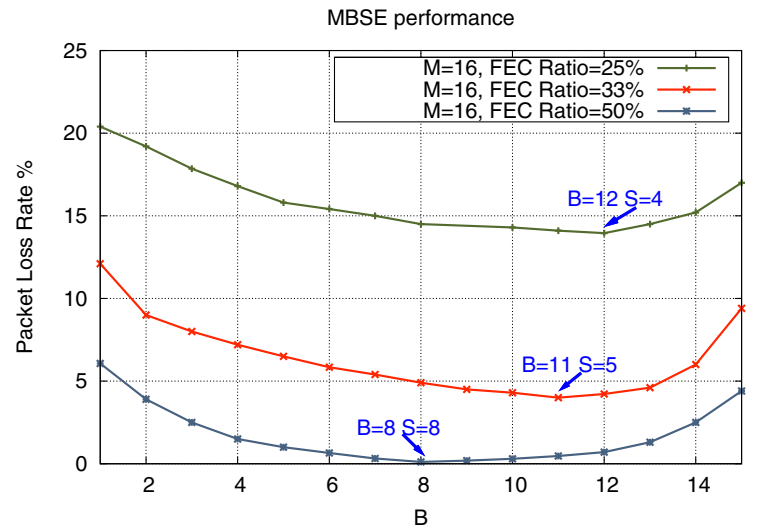

Figure 6. Packet Loss Rate in LMS channel while varying $B$ and $S$ subject to $B+S=16$ for a FEC ratio of $25 \%, 33 \%$ and $50 \%$

analyzed. We have demonstated that there is a realtionship between the FEC ratio and the interleaving parameters. As a result, an analytic method to calculate the optimum parameters of MBSE was derived, furthemore an algorithm was provided. To validate our analytic method, several experiments have been performed for different usage scenarios. Experiments have been done using a real implementation of MBSE in two UDcast standard equiments and a hardware channel emulator implementing two typical mobile channels (TU6 and LMS). Results show that, comparing to the worst case, our recom-

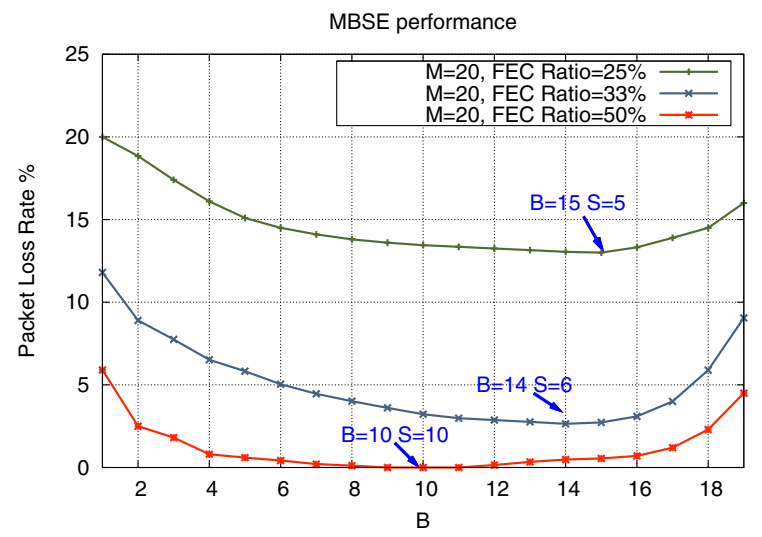

Figure 7. Packet Loss Rate in LMS channel while varying $B$ and $S$ subject to $B+S=20$ for a FEC ratio of $25 \%, 33 \%$ and $50 \%$

mendations for the burst interleaver setup can optimize the recovering capacity by more than 5\% in term of PLR and provide a gain of $\sim 1 \mathrm{db}$ in term of $\mathrm{C} / \mathrm{N}$.

In a future work, we intend to examine the effects of the repetition interval and the encoding matrix size against the packet loss rate in a DVB-SH transmission. We will also study the performance of a two-dimensional FEC scheme (MPEFEC and Multi-burst Sliding Encoding) using a recursive decoding.

\section{REFERENCES}

[1] M. Kornfeld and G. May, "DVB-H and IP Datacast-Broadcast to Handheld Devices," IEEE Trans. Broadcast., vol. 53, no. 1, Mar. 2007.

[2] Transmission System for Handheld Terminals (DVB-H), Digital Video Broadcasting (DVB) Std. ETSI (EN) 302 304, Nov. 2004, release 1.1.1.

[3] Framing Structure, Channel Coding and Modulation for Terrestrial Television, Digital Video Broadcasting (DVB) Std. ETSI (EN) 300 744, Nov. 2004, release 1.5.1.

[4] DVB-H Implementation Guide-lines, Digital Video Broadcasting (DVB) Std. ETSI TR 102 377, Nov. 2005, release 1.2.1.

[5] System Specifications for Satellite Services to Handheld Devices (SH) Below $3 \mathrm{GHz}$, Digital Video Broadcasting (DVB) Working Draft Proposed Standard DVB-SSP0162, Rev. 5, Jan. 2007, release 1.6.2.

[6] IP Datacast over over DVB-H: Phase 1 specifications, Digital Video Broadcasting (DVB) Std. TS 102 468, Nov. 2005, release 1.1.1.

[7] IP Datacast over DVB-H: Electronic Service Guide (ESG), Digital Video Broadcasting (DVB) Std. TS 102 471, Nov. 2006, release 1.2.1.

[8] Mega-frame for Single Frequency Network (SFN) synchronization, Digital Video Broadcasting (DVB) Std. TS 101 191, Jun. 2004, release 1.4.1.

[9] C. Loo and N. Secord, "Computer models for fading channels with applications to digital transmission," IEEE Trans. Veh. Technol., vol. 40, no. 4, pp. 700- 707, Nov. 1991.

[10] G. Faria, J. A. Henriksson, E. Stare, and P. Talmola, "DVB-H: Digital Broadcast Services to Handheld Devices," in Proceedings of the IEEE, vol. 94, no. 1, Jan. 2006, pp. 194- 209.

[11] M. Kornfeld, "Optimizing the DVB-H Time Interleaving Scheme on the Link Layer for High Quality Mobile Broadcasting Reception," in IEEE International Symposium on Consumer Electronics (ISCE), Dallas, USA, Jun. 2007.

[12] UDcast home page. [Online]. Available: http://www.UDcast.com

[13] DVB-SH Implementation Guidelines, Digital Video Broadcasting (DVB) Working Draft Proposed Standard DVB-SSP 0252r9f, Rev. 14, Apr. 2008.

[14] Information Technology-Generic Coding Of Moving Pictures And Associated Audio Information:Systems, International Organization for Standardization (ISO) Std. ISO/IEC 13 818-1, 2000, second edition.

[15] O. Rousset and J. L. Pavy, SSH-2000_DVB-SH Signal Generator Technical Specification, Teamcast, Mar. 2008, release 4.3. 